Georgian Mathematical Journal

Volume 14 (2007), Number 2, 253-267

\title{
ON APPROXIMATION OF THE PERTURBED INCLUSION
}

\author{
ALEXANDER I. BULGAKOV, ANNA A. GRIGORENKO, AND \\ ANATOLIY I. KOROBKO
}

\begin{abstract}
The paper is concerned with the so-called perturbed inclusion in the space of continuous functions. The right-hand side of the inclusion is represented by an algebraic sum of the values of two multi-valued maps, one of which consists of compacts and the other is not necessarily closed-valued and is a composition of a linear integral operator and multimap convexvalued with respect to switching. For such an inclusion it is proved that approximation in the space of summable functions of the values of a multimap convex-valued with respect to switching is not always a stable process. The necessary and sufficient condition for the closure of the set of approximate solutions to converge to the closure of the set of solutions for perturbed inclusion is derived.
\end{abstract}

2000 Mathematics Subject Classification: 34A60, 45G15, $47 \mathrm{H} 04$.

Key words and phrases: Asymptotic representation of the solutions sets for inclusions.

The perturbed inclusion with external perturbations has already been studied in $[1-5]$ in the situation where a multimap $\Delta:[a, b] \times C^{n}[a, b] \rightarrow \operatorname{comp}\left[\mathbb{R}^{n}\right]$ generating a multimap convex-valued with respect to switching (the definition is given below) satisfies the Carathéodory conditions. Perturbations as such appear in different kinds of applications, for they characterize the errors of calculations of the values of the corresponding multimaps. And, as it has been shown in $[1-5]$, these perturbations should not to be ignored because they can lead to considerable changes of the set of solutions for the perturbed inclusion. In this work we deal with the perturbed inclusion in the case where the multimap $\Delta:[a, b] \times C^{n}[a, b] \rightarrow \operatorname{comp}\left[\mathbb{R}^{n}\right]$ is integrably continuous (see the definition below).

Let $X$ be a linear normed space with the norm $\|\cdot\|_{X}$. Denote by $B_{X}[x, \epsilon]$ an open ball in the space $X$ with center $x \in X$ and radius $\epsilon>0$, if $\epsilon=0$, then $B_{X}[x, 0] \equiv x$. Let $U \subset X$. Then $\bar{U}$ stands for the closure of $U$, co $U$ stands for the convex hull of $U, \overline{\operatorname{co}} U=\overline{\operatorname{co} U}$, $\operatorname{ext} U$ denotes the set of all extreme points of $U, \overline{\operatorname{ext}} U=\overline{\operatorname{ext} U} ;\|U\|_{X}=\sup _{x \in U}\|u\|_{X}$. Next, let $\Omega(U)$ be the set of all nonempty closed convex subsets of $U, U^{\epsilon} \equiv \overline{\cup_{u \in U} B[u, \epsilon]}$, if $\epsilon>0$, and $U^{0} \equiv \bar{U}$; $\rho_{X}[x, U]$ is a distance from the point $x \in X$ to the set $U$ in the space $X$; $h_{X}[\cdot ; \cdot]$ is the Hausdorff distance in the space $X$ between the corresponding sets; comp $[X](\operatorname{cl}[X])$ is a set of all nonempty compacts (all nonempty closed bounded subsets) of $X$. 
Let $\mathbb{R}^{n}$ be the $n$-dimensional space of column-vectors with the norm $|\cdot|$. Denote by $C^{n}[a, b],\left(L^{n}[a, b]\right)$ the space of continuous (Lebesgue summable) functions $x:[a, b] \rightarrow \mathbb{R}^{n}$ with the norm $\|x\|_{C^{n}[a, b]}=\max \{|x(t)|: t \in[a, b]\}$ $\left(\|x\|_{L^{n}[a, b]}=\int_{a}^{b}|x(s)| d s\right)$.

Let $\Phi \subset \stackrel{a}{L^{n}}[a, b]$. We say that the set $\Phi$ is convex with respect to switching (decomposable) if for any $x, y \in \Phi$ and every measurable set $\mathcal{U} \subset[a, b]$ the inclusion $\chi(\mathcal{U}) x+\chi([a, b] \backslash \mathcal{U}) y \in \Phi$ takes place, here $\chi(\cdot)$ is the characteristic function of the corresponding set. Denote by $\Pi\left[L^{n}[a, b]\right]$ the set of all closed bounded and convex with respect to switching subsets of $L^{n}[a, b]$.

Throughout the paper the measurability of a single-valued map is considered in the sense of Lebesgue, and the measurability of a multi-valued map in the sense of $[6]$.

Let $F:[a, b] \rightarrow \operatorname{comp}\left[\mathbb{R}^{n}\right]$ be a measurable map. Denote $S(F)=\{y \in$ $L^{n}[a, b]: y(t) \in F(t)$ for a.e. $\left.t \in[a, b]\right\}$.

When $X=\mathbb{R}^{n}$ we, for convenience, omit the index $\mathbb{R}^{n}$ in the notation of the Hausdorff distance.

Consider, in the space $C^{n}[a, b]$, an inclusion

$$
x \in \Psi(x)+V \Phi(x)
$$

where maps $\Psi: C^{n}[a, b] \rightarrow \operatorname{comp}\left[C^{n}[a, b]\right], \Phi: C^{n}[a, b] \rightarrow \Pi\left[L^{n}[a, b]\right]$ are continuous in the Hausdorff sense, the linear integral operator $V: L^{n}[a, b] \rightarrow C^{n}[a, b]$ defined as

$$
(V z)(t)=\int_{a}^{b} V(t, s) z(s) d s, \quad t \in[a, b],
$$

maps every weakly compact set in $L^{n}[a, b]$ into a relatively compact set of the space $C^{n}[a, b]$. We call inclusion (1) a perturbed inclusion (see [1-5]).

Under a solution of inclusion(1) we understand an element $x \in C^{n}[a, b]$ satisfying (1). So, the continuous function $x:[a, b] \rightarrow \mathbb{R}^{n}$ is a solution of inclusion (1) if and only if there exist elements $v \in \Psi(x)$ and $z \in \Phi(x)$ such that equality $x=v+V z$ takes place.

Following [1] we say that a function $x \in C^{n}[a, b]$ is a quasisolution of inclusion (1) if there exist an element $v \in \Psi(x)$ and a sequence

$$
z_{i} \in \Phi(x), \quad i=1,2, \ldots,
$$

such that $x_{i}=v+V z_{i} \rightarrow x$ in the space $C^{n}[a, b]$ as $i \rightarrow \infty$. By $\mathcal{H}$ we denote the set of all quasisolutions for (1).

Consider, in the space $C^{n}[a, b]$, the inclusion

$$
x \in \Psi(x)+V \overline{\operatorname{co}} \Phi(x) .
$$

Following [4], we call inclusion (4), a "convexified" perturbed inclusion. Let $H_{\text {co }}$ be the set of all solutions for (4). 
Theorem 1. Let the linear continuous integral operator $V: L^{n}[a, b] \rightarrow$ $C^{n}[a, b]$ defined by (2) map every weakly compact set in $L^{n}[a, b]$ into a relatively compact set of $C^{n}[a, b]$. Then the equality $H_{\mathrm{co}}=\mathcal{H}$ holds.

Proof. We show first that $H_{\text {co }} \subset \mathcal{H}$. Let $x \in H_{\text {co }}$. Then we can find functions $v \in \Psi(x)$ and $z \in \overline{\operatorname{co}} \Phi(x)$ such that the equality $x=v+V z$ holds. According to [7], for the function $z$ there exists a sequence $z_{i} \in \Phi(x), i=1,2, \ldots$, such that $z_{i} \rightarrow z$ weakly in $L^{n}[a, b]$ as $i \rightarrow \infty$. This means that for any $t \in[a, b]$ and as $i \rightarrow \infty$ we have

$$
y_{i}(t)=v(t)+\left(V z_{i}\right)(t) \rightarrow x(t)=v(t)+(V z)(t) .
$$

Since the sequence $V z_{i}, i=1,2, \ldots$, is compact in $C^{n}[a, b]$, we can say that $y_{i} \rightarrow x$ in $C^{n}[a, b]$ as $i \rightarrow \infty$. Hence, $H_{\text {co }} \subset \mathcal{H}$.

Now, prove that $\mathcal{H} \subset H_{\text {co. Let }} x \in \mathcal{H}$. Then there exist $v \in \Psi(x)$ and a sequence $z_{i} \in L^{n}[a, b]$ satisfying inclusion (3), such that $y_{i}=v+V z_{i} \rightarrow x$ in $C^{n}[a, b]$ as $i \rightarrow \infty$. The sequence $z_{i}$ is weakly compact in $L^{n}[a, b]$, so, without loss of generality, we can say that $z_{i} \rightarrow z$ weakly in $L^{n}[a, b]$ for some $z \in L^{n}[a, b]$ as $i \rightarrow \infty$. Since $z_{i} \in \overline{\mathrm{co}} \Phi(x)$ (see $(3)$ ), we have the inclusion $z \in \overline{\mathrm{co}} \Phi(x)$ (see [6]) and the equality $x=v+V z$, which means that $x \in H_{\text {co }}$ and, consequently, $\mathcal{H} \subset H_{\text {co. }}$

Remark 1 . Note, that Theorem 1 holds without any continuity of $\Phi: C^{n}[a, b] \rightarrow$ $\Pi\left[L^{n}[a, b]\right]$ and $\Psi: C^{n}[a, b] \rightarrow \operatorname{comp}\left[C^{n}[a, b]\right]$.

Let $U$ be a closed subset of $C^{n}[a, b]$. Denote by $\mathcal{H}(U)$ a set of all quasisolutions for inclusion (1) which belong to $U$. We say that $U \subset C^{n}[a, b]$ satisfies property $\mathcal{A}$, if for any $x \in \mathcal{H}(U)$ there exist an element $v \in \Psi(x)$ and a sequence $z_{i} \in$ $L^{n}[a, b], i=1,2, \ldots$, satisfying (3), such that for each $i=1,2, \ldots$ the inclusion $x_{i}=v+V z_{i} \in U$ takes place and $x_{i} \rightarrow x$ in $C^{n}[a, b]$ as $i \rightarrow \infty$.

Note that if the operator defined by the right-hand side of inclusion (1), maps closed convex set $U \subset C^{n}[a, b]$ into itself, then according to Theorem 1 the set $U$ satisfies property $\mathcal{A}$. Moreover, in some cases (for example, in studying periodic or multi-point boundary value problems (see $[5,8,9])$ ) it is easier to show that the set satisfies property $\mathcal{A}$ than to prove that the set is mapped by the corresponding operator into itself).

Let an multi-valued map $\Delta:[a, b] \times C^{n}[a, b] \rightarrow \operatorname{comp}\left[\mathbb{R}^{n}\right]$ possess the following property: for any fixed $x \in C^{n}[a, b]$ the map $\Delta(\cdot, x)$ is measurable and satisfies the inequality

$$
\Phi(x)=\left\{y \in L^{n}[a, b]: y(t) \in \Delta(t, x) \text { for a.e. } t \in[a, b]\right\} .
$$

Such a map does exist (see, e.g., $[1,10]$ ). Since the map $\Phi(x)$ is bounded by a summable function (see $[1,10]$ ), then for any $x \in C^{n}[a, b]$ the map $\Delta(\cdot, x)$ is also bounded by a summable function. By the analogy with the Nemitsky operator, we call the map $\Delta:[a, b] \times C^{n}[a, b] \rightarrow \operatorname{comp}\left[\mathbb{R}^{n}\right]$ defined by equality (5) the map generating the operator $\Phi: C^{n}[a, b] \rightarrow \Pi\left[L^{n}[a, b]\right]$.

We say that the multi-valued map $\Delta:[a, b] \times C^{n}[a, b] \rightarrow$ comp $\left[\mathbb{R}^{n}\right]$ is integrably continuous (continuous in the mean) at the point $x \in C^{n}[a, b]$ if for any sequence 
$x_{i} \in C^{n}[a, b], i=1,2, \ldots$, converging to $x$ in the space $C^{n}[a, b]$ as $i \rightarrow \infty$ the relation

$$
\lim _{i \rightarrow \infty} \int_{a}^{b} h\left[\Delta\left(t, x_{i}\right) ; \Delta(t, x)\right] d t=0
$$

holds. The map $\Delta:[a, b] \times C^{n}[a, b] \rightarrow \operatorname{comp}\left[\mathbb{R}^{n}\right]$ is called integrably continuous (continuous in the mean) on $C^{n}[a, b]$ if it is integrably continuous (continuous in the mean) at every point $x \in C^{n}[a, b]$.

Note that the map $\Delta:[a, b] \times C^{n}[a, b] \rightarrow \operatorname{comp}\left[\mathbb{R}^{n}\right]$ is integrably continuous if and only if the map $\Phi: C^{n}[a, b] \rightarrow \Pi\left[L^{n}[a, b]\right]$ is continuous (see [1]).

By $P\left(C^{n}[a, b] \times[0, \infty)\right)\left(\widetilde{P}\left(C^{n}[a, b] \times[0, \infty)\right)\right)$ we denote the set of all continuous functions $q: C^{n}[a, b] \times[0, \infty) \rightarrow[0, \infty)$, such that for every $x \in C^{n}[a, b]$ the relation $q(x, 0)=0$ holds $\left(q(x, 0)=0\right.$ holds and for any $(x, \delta) \in C^{n}[a, b] \times(0, \infty)$ the inequality $q(x, \delta)>0$ takes place $)$.

Consider now the operator $\Phi: C^{n}[a, b] \rightarrow \Pi\left[L^{n}[a, b]\right]$ and its generating map $\Delta:[a, b] \times C^{n}[a, b] \rightarrow \operatorname{comp}\left[\mathbb{R}^{n}\right]$. The values of the map $\Phi(\cdot)$ and, hence, the values of the operator $\Delta(\cdot, \cdot)$, can be calculated with some accuracy. Let the accuracy of the calculation of the values of the operator $\Phi(\cdot)$ be defined by a function $\eta(\cdot, \cdot) \in P\left(C^{n}[a, b] \times[0, \infty)\right)$, and consider a map $\Phi_{\eta}: C^{n}[a, b] \times$ $[0, \infty) \rightarrow \operatorname{cl}\left[L^{n}[a, b]\right]$ defined as follows

$$
\Phi_{\eta}(x, \delta)=(\Phi(x))^{\eta(x, \delta)},
$$

where the function $\eta(\cdot, \cdot) \in P\left(C^{n}[a, b] \times[0, \infty)\right)$ at every point $x \in C^{n}[a, b]$ for each fixed $\delta \in[0, \infty)$ defines the calculation error of the value of $\Phi(\cdot)$. Next, we call the function $\eta(\cdot, \cdot)$ the radius of external perturbations of the map $\Phi(\cdot)$ or simply the radius of external perturbations. From (6) it follows that

$$
h_{L^{n}[a, b]}\left[\Phi(x) ; \Phi_{\eta}(x)\right]=\eta(x, \delta) .
$$

From equality (7) one can get

$$
\lim _{\delta \rightarrow 0+0} h_{L^{n}[a, b]}\left[\Phi(x) ; \Phi_{\eta}(x)\right]=0 .
$$

So, depending on the radius of external perturbations $\eta(\cdot, \cdot) \in P\left(C^{n}[a, b] \times\right.$ $[0, \infty))$, all maps $\Phi_{\eta}: C^{n}[a, b] \rightarrow \operatorname{cl}\left[L^{n}[a, b]\right]$ of form (6) are close to the map $\Phi: C^{n}[a, b] \rightarrow \Pi\left[L^{n}[a, b]\right]$ in the sense of (8). We call this approximation of the operator $\Phi(\cdot)$ the approximation by embedding in the mean or, simply, the approximation in the mean, while the map $\Phi_{\eta}: C^{n}[a, b] \times[0, \infty) \rightarrow \operatorname{cl}\left[L^{n}[a, b]\right]$ the approximating operator.

Let $U$ be a nonempty closed convex set of the space $C^{n}[a, b]$ and let $\omega(\cdot, \cdot) \in$ $\widetilde{P}\left(C^{n}[a, b] \times[0, \infty)\right)$. Consider a multi-valued map $M_{U}(\omega): U \times[0, \infty) \rightarrow \Omega(U)$ defined as

$$
M_{U}(\omega)(x, \delta)=\overline{B_{C^{n}[a, b]}[x, \omega(x, \delta)]} \cap U .
$$

Lemma 1. Let $U$ be a nonempty closed convex set of the space $C^{n}[a, b]$ and let $\omega(\cdot, \cdot) \in \widetilde{P}\left(C^{n}[a, b] \times[0, \infty)\right)$. Then the map $M_{U}(\omega): U \times[0, \infty) \rightarrow \Omega(U)$ defined by (9) is continuous in the Hausdorff sense. 
Indeed, according to the definition of the map $M_{U}(\omega)(\cdot, \cdot)$ and to [1], for any $x, y \in U$ and $\delta_{1}, \delta_{2} \geq 0$ we have

$$
h_{C^{n}[a, b]}\left[M_{U}(\omega)\left(x, \delta_{1}\right) ; M_{U}(\omega)\left(y, \delta_{2}\right)\right] \leq\|x-y\|_{C^{n}[a, b]}+\left|\omega\left(x, \delta_{1}\right)-\omega\left(y, \delta_{2}\right)\right| .
$$

So, the map $M_{U}(\omega)(\cdot, \cdot)$ is continuous in the Hausdorff sense.

Define now a map $\varphi_{U}(\omega): U \times[0, \infty) \rightarrow[0, \infty)$ as follows

$$
\varphi_{U}(\omega)(x, \delta)=\sup _{y \in M_{U}(\omega)(x, \delta)} h_{L^{n}[a, b]}[\Phi(x) ; \Phi(y)]
$$

here the map $M_{U}(\omega): U \times[0, \infty) \rightarrow \Omega(U)$ is defined by equality (9).

The value of the function $\varphi_{U}(\omega)(\cdot, \cdot)$ at $(x, \delta) \in U \times[0, \infty)$ is called the modulus of continuity of the map $\Phi: C^{n}[a, b] \rightarrow \Pi\left[L^{n}[a, b]\right]$ at the point $(x, \delta)$ on the set $\overline{B_{C^{n}[a, b]}[x, \omega(x, \delta)]} \cap U$, the function $\omega(\cdot, \cdot)$ is called the function of radius of the modulus of continuity or, simply, the radius of continuity, and the function $\varphi_{U}(\omega)(\cdot, \cdot)$ is called a function of the modulus of continuity of the map $\Phi: C^{n}[a, b] \rightarrow \Pi\left[L^{n}[a, b]\right]$ on the set $U$ with respect to the radius of continuity $\omega(\cdot, \cdot)$ or, simply, modulus of continuity.

Remark 2. If the multi-valued map $\Delta:[a, b] \times C^{n}[a, b] \rightarrow \operatorname{comp}\left[\mathbb{R}^{n}\right]$ generating the operator $\Phi: C^{n}[a, b] \rightarrow \Pi\left[L^{n}[a, b]\right]$ is known, then by analogy one can define the mean (integral) modulus of continuity $\widetilde{\varphi}_{U}(\omega): U \times[0, \infty) \rightarrow[0, \infty)$ of the map with by equality

$$
\widetilde{\varphi}_{U}(\omega)(x, \delta)=\sup _{y \in M_{U}(\omega)(x, \delta)} \int_{a}^{b} h[\Delta(t, x) ; \Delta(t, y)] d t .
$$

Along with this, the modulus of continuity of the operator $\Phi: C^{n}[a, b] \rightarrow$ $\Pi\left[L^{n}[a, b]\right]$, as well as the mean modulus of continuity of the map $\Delta:[a, b] \times$ $C^{n}[a, b] \rightarrow \operatorname{comp}\left[\mathbb{R}^{n}\right]$ satisfy the relations (see $[1]$ )

$$
\varphi_{U}(\omega)(x, \delta) \leq \widetilde{\varphi}_{U}(\omega)(x, \delta) \leq 2 \varphi_{U}(\omega)(x, \delta)
$$

for any $(x, \delta) \in U \times[0, \infty)$.

Lemma 2. Let $U$ be a nonempty compact convex set of the space $C^{n}[a, b]$ and let $\omega(\cdot, \cdot) \in P\left(C^{n}[a, b] \times[0, \infty)\right)$. Then the maps $\varphi_{U}(\omega): U \times[0, \infty) \rightarrow$ $[0, \infty), \widetilde{\varphi}_{U}(\omega)(x, \delta): U \times[0, \infty) \rightarrow[0, \infty)$ defined by $(10),(11)$, respectively, are continuous and for any $x \in U$ the following relations take place:

$$
\lim _{\substack{z \rightarrow x \\ \delta \rightarrow 0+0}} \varphi_{U}(\omega)(z, \delta)=0, \quad \lim _{\substack{z \rightarrow x \\ \delta \rightarrow 0+0}} \widetilde{\varphi}_{U}(\omega)(z, \delta)=0 .
$$

Proof. We show first that the function $\varphi_{U}(\omega)(\cdot, \cdot)$ is upper semicontinuous. Let the sequences $x_{i}(\in U) \rightarrow x$ in $C^{n}[a, b]$ and $\delta_{i}(\in[0, \infty)) \rightarrow \delta(\in[0, \infty))$. Take $\varepsilon>0$ and let the functions $y_{i} \in M_{U}(\omega)\left(x_{i}, \delta_{i}\right)$ satisfy the inequality

$$
\varphi_{U}(\omega)(x, \delta) \leq h_{L^{n}[a, b]}\left[\Phi(x), \Phi\left(y_{i}\right)\right]+\varepsilon
$$

for each $i=1,2, \ldots$. Without loss of generality suppose that $y_{i} \rightarrow y$ in $C^{n}[a, b]$ as $i \rightarrow \infty$. From Lemma 1 we get the inclusion $y \in M_{U}(\omega)(x, \delta)$ from which, 
according to (13), it follows that for any $i=1,2, \ldots$

$$
\varphi_{U}(\omega)\left(x_{i}, \delta_{i}\right) \leq h_{L^{n}[a, b]}\left[\Phi\left(y_{i}\right) ; \Phi(y)\right]+h_{L^{n}[a, b]}[\Phi(y) ; \Phi(x)]+\varepsilon .
$$

So, for every $i=1,2, \ldots$ the relation

$$
\varphi_{U}(\omega)\left(x_{i}, \delta_{i}\right) \leq h_{L^{n}[a, b]}\left[\Phi\left(y_{i}\right) ; \Phi(y)\right]+\varphi_{U}(\omega)(x, \delta)+\varepsilon
$$

takes place. Passing in the last inequality to the limit as $i \rightarrow \infty$ we get

$$
\varlimsup_{i \rightarrow \infty} \varphi_{U}(\omega)\left(x_{i}, \delta_{i}\right) \leq \varphi_{U}(\omega)(x, \delta) .
$$

We prove now that $\varphi_{U}(\omega)(\cdot, \cdot)$ is lower semicontinuous. Indeed, let the sequences $\left\{x_{i}\right\}$ and $\left\{\delta_{i}\right\}$ be taken as previously. Let $\varepsilon>0$ and let the function $y \in M_{U}(\omega)(x, \delta)$ satisfy the inequality

$$
\varphi_{U}(\omega)(x, \delta) \leq h_{L^{n}[a, b]}[\Phi(x) ; \Phi(y)]+\varepsilon .
$$

According to Lemma 1 , there exists a sequence $y_{i} \in M_{U}(\omega)\left(x_{i}, \delta_{i}\right), i=1,2, \ldots$, such that $y_{i} \rightarrow y$ in $C^{n}[a, b]$ as $i \rightarrow \infty$. Then from (14) we have the estimation $\varphi_{U}(\omega)(x, \delta) \leq h_{L^{n}[a, b]}\left[\Phi(x) ; \Phi\left(x_{i}\right)\right]+h_{L^{n}[a, b]}\left[\Phi\left(x_{i}\right) ; \Phi\left(y_{i}\right)\right]+h_{L^{n}[a, b]}\left[\Phi\left(y_{i}\right) ; \Phi(y)\right]+\varepsilon$.

Hence, for every $i=1,2, \ldots$ the relation

$$
\varphi_{U}(\omega)(x, \delta) \leq h_{L^{n}[a, b]}\left[\Phi(x) ; \Phi\left(x_{i}\right)\right]+\varphi_{U}(\omega)\left(x_{i}, \delta_{i}\right)+h_{L^{n}[a, b]}\left[\Phi\left(y_{i}\right) ; \Phi(y)\right]+\varepsilon
$$

takes place. Passing to a limit we get

$$
\varphi_{U}(\omega)(x, \delta) \leq \lim _{i \rightarrow \infty} \varphi_{U}(\omega)\left(x_{i}, \delta_{i}\right)
$$

So, the function $\varphi_{U}(\omega)(\cdot, \cdot)$ is continuous by both arguments.

By analogy one can show that the function $\widetilde{\varphi}_{U}(\omega)(\cdot, \cdot)$ is continuous.

Next, since for any $x \in U$ the equalities $\varphi_{U}(\omega)(x, 0)=\widetilde{\varphi}_{U}(\omega)(x, 0)=0$ take place, relations (12) follow immediately from the continuity of the functions $\varphi_{U}(\omega)(\cdot, \cdot), \widetilde{\varphi}_{U}(\omega)(\cdot, \cdot)$. The statement is proved.

Let $U$ be a nonempty closed set of the space $C^{n}[a, b]$. We say that a function $\eta(\cdot, \cdot) \in P\left(C^{n}[a, b] \times[0, \infty)\right)$ estimates from above - uniformly on the set $U \subset$ $C^{n}[a, b]$ - the modulus of continuity of the operator $\Phi: C^{n}[a, b] \rightarrow \Pi\left[L^{n}[a, b]\right]$, (the mean modulus of continuity of the map $\Delta:[a, b] \times C^{n}[a, b] \rightarrow \operatorname{comp}\left[\mathbb{R}^{n}\right]$ ) with respect to the radius of continuity $\omega(\cdot, \cdot) \in \widetilde{P}\left(C^{n}[a, b] \times[0, \infty)\right)$ if for every $\varepsilon>0$ there exists $\delta(\varepsilon)>0$ such that for every $x \in U$ and $\delta \in[0, \delta(\varepsilon))$ the estimation

$$
\varphi_{U}(\omega)(x, \delta) \leq \eta(x, \varepsilon), \quad\left(\widetilde{\varphi}_{U}(\omega)(x, \delta) \leq \eta(x, \varepsilon)\right)
$$

takes place, where the maps $\varphi_{U}(\omega): U \times[0, \infty) \rightarrow[0, \infty), \widetilde{\varphi}_{U}(\omega): U \times[0, \infty) \rightarrow$ $[0, \infty)$ are defined by $(10),(11)$, respectively.

Let $U \subset C^{n}[a, b]$ and $\omega(\cdot, \cdot) \in P\left(C^{n}[a, b] \times[0, \infty)\right)$. Define the functions $\lambda_{U}(\omega):[0, \infty) \rightarrow[0, \infty), \widetilde{\lambda}_{U}(\omega):[0, \infty) \rightarrow[0, \infty)$ as follows

$$
\lambda_{U}(\omega)(\delta)=\sup _{x \in U} \varphi_{U}(\omega)(x, \delta), \quad \widetilde{\lambda}_{U}(\omega)(\delta)=\sup _{x \in U} \widetilde{\varphi}_{U}(\omega)(x, \delta)
$$


Lemma 3. Let $U$ be a nonempty compact convex set in the space $C^{n}[a, b]$ and let $\omega(\cdot, \cdot) \in P\left(C^{n}[a, b] \times[0, \infty)\right)$. Then the maps $\lambda_{U}(\omega):[0, \infty) \rightarrow[0, \infty)$, $\widetilde{\lambda}_{U}(\omega):[0, \infty) \rightarrow[0, \infty)$ defined by $(15)$ are continuous and satisfy the equalities $\lambda_{U}(\omega)(0)=\widetilde{\lambda}_{U}(\omega)(0)=0$.

Proof. Show that the function $\lambda_{U}(\omega)(\cdot)$ is upper semicontinuous. Indeed, let $\delta_{i}(\in[0, \infty)) \rightarrow \delta$ as $i \rightarrow \infty$ and $x \in U$ satisfy the equality $\lambda_{U}(\omega)(\delta)=$ $\varphi_{U}(\omega)(x, \delta)$. Since for every $i=1,2, \ldots$ the relation

$$
\lambda_{U}(\omega)\left(\delta_{i}\right) \geq \varphi_{U}(\omega)\left(x, \delta_{i}\right)
$$

takes place, from the continuity of the map $\varphi_{U}(\omega)(\cdot, \cdot)$ it follows that

$$
\varlimsup_{i \rightarrow \infty} \lambda_{U}(\omega)\left(\delta_{i}\right) \geq \varphi_{U}(\omega)(x, \delta) .
$$

So, the map $\lambda_{U}(\omega)(\cdot)$ is upper semicontinuous.

Next, prove that $\lambda_{U}(\omega)(\cdot)$ is lower semicontinuous. Let the sequence $\delta_{i}(\in$ $[0, \infty)), i=1,2, \ldots$ converge to $\delta \geq 0$ and let the equality $\lambda_{U}(\omega)\left(\delta_{i}\right)=$ $\varphi_{U}(\omega)\left(x_{i}, \delta_{i}\right)$ hold for some element $x_{i} \in U, i=1,2, \ldots$ Without loss of generality suppose that $x_{i} \rightarrow x(\in U)$ in $C^{n}[a, b]$ as $i \rightarrow \infty$. Then we get

$$
\lim _{i \rightarrow \infty} \lambda_{U}(\omega)\left(\delta_{i}\right)=\lim _{i \rightarrow \infty} \varphi_{U}(\omega)\left(x_{i}, \delta_{i}\right)=\varphi_{U}(\omega)(x, \delta) \leq \lambda_{U}(\omega)(\delta)
$$

So, the function $\lambda_{U}(\omega)(\cdot)$ is lower semicontinuous, and, hence, continuous. By analogy one can show that the map $\widetilde{\lambda}_{U}(\omega):[0, \infty) \rightarrow[0, \infty)$ is continuous.

The relations $\lambda_{U}(\omega)(0)=\widetilde{\lambda}_{U}(\omega)(0)=0$ immediately follow from the definitions of the maps $\lambda_{U}(\omega)(\cdot), \tilde{\lambda}_{U}(\omega)(\cdot)$.

Corollary 1. Let $U$ be a nonempty compact convex set in the space $C^{n}[a, b]$ and let $\omega(\cdot, \cdot) \in \widetilde{P}\left(C^{n}[a, b] \times[0, \infty)\right)$. Then the function $\lambda_{U}(\omega)(\cdot)\left(\widetilde{\lambda}_{U}(\omega)(\cdot)\right) \in$ $P\left(C^{n}[a, b] \times[0, \infty)\right)$ defined by (15) estimates from above - uniformly on the set $U \subset C^{n}[a, b]$ - the modulus of continuity of the map $\Phi: C^{n}[a, b] \rightarrow \Pi\left[L^{n}[a, b]\right]$ (the mean modulus of continuity of the map $\Delta:[a, b] \times C^{n}[a, b] \rightarrow \operatorname{comp}\left[\mathbb{R}^{n}\right]$ ) with respect to the radius of continuity $\omega(\cdot, \cdot)$.

Remark 3 . Note that by changing the radius of continuity $\omega(\cdot, \cdot)$ one can make the modulus of continuity of the operator $\Phi: C^{n}[a, b] \rightarrow \Pi\left[L^{n}[a, b]\right]$ and the mean modulus of continuity of the map $\Delta:[a, b] \times C^{n}[a, b] \rightarrow \operatorname{comp}\left[\mathbb{R}^{n}\right]$ sufficiently small at every point $(x, \delta) \in U \times(0, \infty)$. This means that the functions $\lambda_{U}(\omega)(\cdot), \widetilde{\lambda}_{U}(\omega)(\cdot)$, defined by $(15)$ may be infinitesimal at every point $\delta>0$.

Let $(\eta(\cdot, \cdot), \xi(\cdot, \cdot)) \in P\left(C^{n}[a, b] \times[0, \infty)\right) \times \widetilde{P}\left(C^{n}[a, b] \times[0, \infty)\right)$. In the space $C^{n}[a, b]$, for every $\delta>0$ consider the inclusion

$$
x \in(\Psi(x))^{\xi(x, \delta)}+V \Phi_{\eta}(x, \delta),
$$

where the map $\Phi_{\eta}: C^{n}[a, b] \times[0, \infty) \rightarrow \operatorname{cl}\left[L^{n}[a, b]\right]$ is defined by (6).

We call the functions $\eta(\cdot, \cdot), \xi(\cdot, \cdot)$ in $(16)$ the external perturbations characterizing the calculation errors of the values of the maps $\Phi: C^{n}[a, b] \rightarrow \Pi\left[L^{n}[a, b]\right]$ 
and $\Psi: C^{n}[a, b] \rightarrow \operatorname{comp}\left[C^{n}[a, b]\right]$. And we call inclusion (16) the inclusion with external perturbations.

We call every solution of (16) for a fixed $\delta>0$ a $\delta$-solution (approximate solution) of inclusion (1). Denote by $H_{\xi(\delta), \eta(\delta)}(U)$ a set of all $\delta$-solutions (approximate solutions) of inclusion (1), which belong to the set $U \subset C^{n}[a, b]$, and by $H(U)$ and $H_{\mathrm{co}}(U)$ the sets of solutions for (1) and (4), respectively, belonging to the set $U \subset C^{n}[a, b]$.

Theorem 2. Let $U$ be a nonempty closed set of the space $C^{n}[a, b]$ such that $U$ satisfies property $\mathcal{A}$ and let $\xi(\cdot, \cdot), \omega(\cdot, \cdot) \in \widetilde{P}\left(C^{n}[a, b] \times[0, \infty)\right)$. Then for any function $\eta(\cdot, \cdot) \in P\left(C^{n}[a, b] \times[0, \infty)\right)$ which estimates from above - uniformly on the set $U \subset C^{n}[a, b]$ - the modulus of continuity of the map $\Phi: C^{n}[a, b] \rightarrow$ $\Pi\left[L^{n}[a, b]\right]$ with respect to the radius of continuity $\omega(\cdot, \cdot) \in \widetilde{P}\left(C^{n}[a, b] \times[0, \infty)\right)$ the following equality takes place

$$
H_{\mathrm{co}}(U)=\bigcap_{\delta>0} \overline{H_{\xi(\delta), \eta(\delta)}(U)}
$$

here $\overline{H_{\xi(\delta), \eta(\delta)}(U)}$ is the closure of the set $H_{\xi(\delta), \eta(\delta)}(U)$ in the space $C^{n}[a, b]$.

Proof. We notice first that since the set $U$ is closed, the set $H_{\text {co }}(U)$, according to [1], is also closed in $C^{n}[a, b]$.

Prove the relation

$$
H_{\mathrm{co}}(U) \subset \bigcap_{\delta>0} \overline{H_{\xi(\delta), \eta(\delta)}(U)} .
$$

Let $x \in H_{\mathrm{co}}(U)$. Show that $x$ is a limit point of the set $H_{\xi(\delta), \eta(\delta)}(U)$ for every $\delta>0$. In accordance with Theorem $1, x$ is a quasisolution of inclusion (1). This means that there exist an element $v \in \Psi(x)$ and a sequence $z_{i} \in \Phi(x)$, $i=1,2, \ldots$ such that the sequence $x_{i}=v+V z_{i} \rightarrow x$ in $C^{n}[a, b]$ as $i \rightarrow \infty$, moreover, due to the property $\mathcal{A}$, we can suppose that $x_{i} \in U$ for each $i=$ $1,2, \ldots$ Next, let $\eta(\cdot, \cdot) \in P\left(C^{n}[a, b] \times[0, \infty)\right)$ estimate from above - uniformly on the set $U$ - the modulus of continuity of the map $\Phi(\cdot)$ with respect to the radius of continuity $\omega(\cdot, \cdot) \in \widetilde{P}\left(C^{n}[a, b] \times[0, \infty)\right)$. Then one can find an $i_{1}$ such that for all $i \geq i_{1}$ the inequality $\left\|x-x_{i}\right\|<\omega\left(x_{i}, \delta\right)$ is true. This means that for all $i \geq i_{1}$ the relation $x \in \overline{B_{C^{n}[a, b]}\left[x_{i}, \omega\left(x_{i}, \delta\right)\right]}$ takes place. So, $x \in M_{U}(\omega)\left(x_{i}, \delta\right)$ for any $i \geq i_{1}$.

Further, from the definition of the uniform estimation from above of the modulus of continuity of the set $\Phi(\cdot)$ it follows that there exists a number $i_{2} \geq i_{1}$ such that for any $i \geq i_{2}$ the inequality

$$
\varphi_{U}(\omega)\left(x_{i},\left\|x-x_{i}\right\|_{C^{n}[a, b]}\right) \leq \eta\left(x_{i}, \delta\right)
$$

holds. From (19) for every $i \geq i_{2}$ we get

$$
\begin{aligned}
\rho_{L^{n}[a, b]}\left[z_{i} ; \Phi\left(x_{i}\right)\right] & \leq h_{L^{n}[a, b]}\left[\Phi(x) ; \Phi\left(x_{i}\right)\right] \\
& \leq \varphi_{U}(\omega)\left(x_{i},\left\|x-x_{i}\right\|_{C^{n}[a, b]}\right) \leq \eta\left(x_{i}, \delta\right) .
\end{aligned}
$$


Next, since $\xi\left(x_{i}, \delta\right) \rightarrow \xi(x, \delta)>0$ as $i \rightarrow \infty$, then one can find $i_{3} \geq i_{2}$ such that for any $i \geq i_{3}$ the following condition is satisfied

$$
h_{C^{n}[a, b]}\left[\Psi(x) ; \Psi\left(x_{i}\right)\right] \leq \xi\left(x_{i}, \delta\right) .
$$

From (20) and (21) it follows that for every $i \geq i_{3}$ we have the inclusion $x_{i} \in$ $H_{\xi(\delta), \eta(\delta)}(U)$, which means that $x$ is a limit point of the set $H_{\xi(\delta), \eta(\delta)}(U)$. So, $x \in \bigcap_{\delta>0} \overline{H_{\xi(\delta), \eta(\delta)}(U)}$ and, hence, relation (18) is proved.

Now we show that

$$
\bigcap_{\delta>0} \overline{H_{\xi(\delta), \eta(\delta)}(U)} \subset H_{\mathrm{co}}(U) .
$$

Let $x \in \bigcap_{\delta>0} \overline{H_{\xi(\delta), \eta(\delta)}(U)}$. Then, for every $i=1,2, \ldots$ there exists $x_{i} \in$ $H_{\xi\left(\frac{1}{i}\right), \eta\left(\frac{1}{i}\right)}(U)$, for which we have the estimation $\left\|x-x_{i}\right\|_{C^{n}[a, b]}<\frac{1}{i}$. Let for each $i=1,2, \ldots$ the functions $v_{i} \in \Psi\left(x_{i}\right)^{\xi\left(x_{i}, \frac{1}{i}\right)}, z_{i} \in \Phi_{\eta}\left(x_{i}, \frac{1}{i}\right)$ be such that

$$
x_{i}=v_{i}+V z_{i}
$$

Next, let the functions $\widetilde{v}_{i} \in \Psi(x), \widetilde{z}_{i} \in \Phi(x), i=1,2, \ldots$, satisfy the equalities

$$
\begin{aligned}
\left\|v_{i}-\widetilde{v}_{i}\right\|_{C^{n}[a, b]} & =\rho_{C^{n}[a, b]}\left[v_{i} ; \Psi(x)\right], \\
\left\|z_{i}-\widetilde{z}_{i}\right\|_{L^{n}[a, b]} & =\rho_{L^{n}[a, b]}\left[z_{i} ; \Phi(x)\right] .
\end{aligned}
$$

Since (24) implies that

$$
\begin{aligned}
\left\|v_{i}-\widetilde{v}_{i}\right\|_{C^{n}[a, b]} & \leq h_{C^{n}[a, b]}\left[\Psi\left(x_{i}\right)^{\xi\left(x_{i}, \frac{1}{i}\right)} ; \Psi(x)\right] \\
& \leq \xi\left(x_{i}, \frac{1}{i}\right)+h_{C^{n}[a, b]}\left[\Psi\left(x_{i}\right) ; \Psi(x)\right]
\end{aligned}
$$

for every $i=1,2, \ldots$, from (26) it follows that

$$
\lim _{i \rightarrow \infty}\left\|v_{i}-\widetilde{v}_{i}\right\|_{C^{n}[a, b]}=0
$$

The set $\Psi(x)$ is compact, then, without loss of generality, one can say that $\widetilde{v}_{i} \rightarrow v \in \Psi(x)$ in the space $C^{n}[a, b]$ as $i \rightarrow \infty$. So, from (27) we get $v_{i} \rightarrow v$ in $C^{n}[a, b]$ as $i \rightarrow \infty$.

Prove now that

$$
\lim _{i \rightarrow \infty}\left\|z_{i}-\widetilde{z}_{i}\right\|_{L^{n}[a, b]}=0 .
$$

Indeed, since from equality (25) for every $i=1,2, \ldots$ we have

$$
\left\|z_{i}-\widetilde{z}_{i}\right\|_{L^{n}[a, b]} \leq h_{L^{n}[a, b]}\left[\Phi_{\eta}\left(x_{i}, \frac{1}{i}\right) ; \Phi(x)\right] \leq \eta\left(x, \frac{1}{i}\right)+h_{L^{n}[a, b]}\left[\Phi\left(x_{i}\right) ; \Phi(x)\right]
$$

from the last estimations we get relation (28).

Next, since the sequence $\widetilde{z}_{i}, i=1,2, \ldots$, is a weakly relatively compact set of the space $L^{n}[a, b]$, we assume, without loss of generality, that $\widetilde{z}_{i} \rightarrow z$ weakly as $i \rightarrow \infty$. According to [6], $z \in \overline{\mathrm{co}} \Phi(x)$. From equality (28) it follows that $z_{i} \rightarrow z$ weakly in $L^{n}[a, b]$ as $i \rightarrow \infty$. Hence, without loss of generality, one can say that $V z_{i} \rightarrow V z$ in $C^{n}[a, b]$ as $i \rightarrow \infty$. So, passing to the limit in $(23)$ as $i \rightarrow \infty$, we get the relation $x=v+V z$, where $v \in \Psi(x), z \in \overline{\mathrm{co}} \Phi(x)$, i.e., $x \in H_{\mathrm{co}}(U)$, 
and, consequently, embedding (22) is true. Equality (17) follows from (18) and $(22)$.

From Remark 2 follows

Corollary 2. Let $U$ be a nonempty closed set in the space $C^{n}[a, b]$ satisfying the property $\mathcal{A}$ and let $\xi(\cdot, \cdot), \omega(\cdot, \cdot) \in \widetilde{P}\left(C^{n}[a, b] \times[0, \infty)\right)$. Then for any function $\eta(\cdot, \cdot) \in P\left(C^{n}[a, b] \times[0, \infty)\right)$ estimating from above - uniformly on the set $U \subset$ $C^{n}[a, b]$ - the mean modulus of continuity of the map $\Delta:[a, b] \times C^{n}[a, b] \rightarrow$ $\operatorname{comp}\left[\mathbb{R}^{n}\right]$ with respect to the radius of continuity $\omega(\cdot, \cdot) \in P\left(C^{n}[a, b] \times[0, \infty)\right)$ the equality (17) takes place.

Remark 4. Note that differential inclusions are a particular case of perturbed inclusions. Since for differential inclusions the equality $\overline{H(U)}=H_{\mathrm{co}}(U)(\overline{H(U)}$ is a closure of the set $H(U)$ in the space $\left.C^{n}[a, b]\right)$ may not be true (see, e.g., $[11,12])$, from Theorem 2 it follows that the relation

$$
\overline{\bigcap_{\delta>0} H_{\xi(\delta), \eta(\delta)}(U)}=\bigcap_{\delta>0} \overline{H_{\xi(\delta), \eta(\delta)}(U)}
$$

may not hold for some $\eta(\cdot, \cdot), \xi(\cdot, \cdot) \in P\left(C^{n}[a, b] \times[0, \infty)\right)$.

Let $U \subset C^{n}[a, b]$. By analogy with $[1,13]$ we say that for inclusion (1) the density principle (density condition) holds on the set $U$ if the following equality holds

$$
\overline{H(U)}=H_{\mathrm{co}}(U),
$$

here $\overline{H(U)}$ is the closure of the set $H(U)$ in the space $C^{n}[a, b]$.

Note that if the density principle holds on some closed set $U \subset C^{n}[a, b]$, then $U$ satisfies the property $\mathcal{A}$.

Remark 5. As has been said previously, the density principle not always takes place. There is an example due to A. Plis (see $[11,12]$ ) which demonstrates this. The first sufficient conditions for equality (29) to be true was derived by A. F. Filippov for a Cauchy problem of the differential inclusion (see [12, 14-18]). For periodic solutions and boundary value problems these conditions are studied in $[19,20]$.

Theorem 3. Let $\xi(\cdot, \cdot) \in \widetilde{P}\left(C^{n}[a, b] \times[0, \infty)\right)$. If $U$ is a nonempty closed set of the space $C^{n}[a, b]$, then for the equality

$$
\overline{H(U)}=\bigcap_{\delta>0} \overline{H_{\xi(\delta), \eta(\delta)}(U)}
$$

to be true for any radius of external perturbations $\eta(\cdot, \cdot) \in P\left(C^{n}[a, b] \times[0, \infty)\right)$ it is sufficient, and for nonempty compact convex $U$ it is also necessary that the density principle hold on the set $U \subset C^{n}[a, b]$.

Proof. We prove first the sufficiency. Let equality (29) be true on the set $U \subset C^{n}[a, b]$. Show that $(30)$ holds for $(\xi(\cdot, \cdot), \eta(\cdot, \cdot)) \in \widetilde{P}\left(C^{n}[a, b] \times[0, \infty)\right) \times$ 
$P\left(C^{n}[a, b] \times[0, \infty)\right)$. Indeed, from the definition of inclusion (16) it follows that for any $\delta>0$ we have the relation

$$
H(U) \subset H_{\xi(\delta), \eta(\delta)}(U) .
$$

Then for every $\delta>0$ the inclusion $\overline{H(U)} \subset \overline{H_{\xi(\delta), \eta(\delta)}(U)}$ takes place, and, hence, we get $\overline{H(U)} \subset \bigcap_{\delta>0} \overline{H_{\xi(\delta), \eta(\delta)}(U)}$.

Show the reverse, i.e., the embedding $\bigcap \overline{H_{\xi(\delta), \eta(\delta)}(U)} \subset \overline{H(U)}$ which, according to equality (29), is equivalent to $\bigcap_{\delta>0} \frac{\delta>0}{H_{\xi(\delta), \eta(\delta)}(U)} \subset H_{\mathrm{co}}(U)$. The last relation can be proved as that was done in the proof of Theorem 2 .

If $U$ is a nonempty compact convex set, then the necessity of equality (30) to be true follows from Theorem 2 and Corollary 1.

Remark 6. Note that having equality (30) for any external perturbations $\eta(\cdot, \cdot), \xi(\cdot, \cdot) \in P\left(C^{n}[a, b] \times[0, \infty)\right)$ is the property of stability of the solutions set $H(U)$ of (1) with respect to these perturbations (see [21]).

Remark 7. Note that from the proof of Theorem 2 it follows that if the density principle holds on set $U \subset C^{n}[a, b]$, then the equality

$$
\bigcap_{\delta>0} \overline{H_{\xi(\delta), \eta(\delta)}(U)}=\bigcap_{\delta>0} \overline{H_{\xi(\delta), \eta(\delta)}\left(U^{\delta}\right)}
$$

is true for any external perturbations $\eta(\cdot, \cdot), \xi(\cdot, \cdot) \in P\left(C^{n}[a, b] \times[0, \infty)\right)$, i.e., the set $\overline{H(U)}$ can be derived through the sets of approximate solutions taking into account the changes of the set to which the solutions belong, as well as without counting those. In other words, "rebuilding" the set $\overline{H(U)}$ by means of sets of approximate solutions in this case does not depend on the " $\delta$-extension" of the set $U$.

As it has been mentioned before, the external perturbations in (16) characterize the calculation error of the values of the multimaps $\Psi: C^{n}[a, b] \rightarrow$ $\operatorname{comp}\left[C^{n}[a, b]\right]$ and $\Phi: C^{n}[a, b] \rightarrow \Pi\left[L^{n}[a, b]\right]$. At the same time, every solution $x:[a, b] \rightarrow \mathbb{R}^{n}$ of inclusion (1) can be calculated in those multumaps with some accuracy degree which can be defined by a function from the set $\widetilde{P}\left(C^{n}[a, b] \times[0, \infty)\right)$. This inaccuracy in finding solutions can be taken into account by means of internal perturbations. Further it will be shown that internal perturbations can affect considerably the solutions set for (1).

Let $U$ be a closed convex set of the space $C^{n}[a, b]$ and let $\xi(\cdot, \cdot), \eta(\cdot, \cdot), \varkappa(\cdot, \cdot) \in$ $P\left(C^{n}[a, b] \times[0, \infty)\right), \sigma(\cdot, \cdot), \theta(\cdot, \cdot), \nu(\cdot, \cdot) \in \widetilde{P}\left(C^{n}[a, b] \times[0, \infty)\right)$. For defining an approximate solution for (1), taking care of internal and external perturbations, we consider the multimaps $\Psi_{\xi, \sigma}: U \times[0, \infty) \rightarrow \operatorname{cl}\left[C^{n}[a, b]\right], \Phi_{\eta, \theta}: U \times[0, \infty) \rightarrow$ $\operatorname{cl}\left[L^{n}[a, b]\right]$ defined as

$$
\begin{aligned}
& \Psi_{\xi, \sigma}(x, \delta)=\left(\Psi\left(M_{U}(\sigma)(x, \delta)\right)\right)^{\xi(x, \delta)} \\
& \Phi_{\eta, \theta}(x, \delta)=\left(\Phi\left(M_{U}(\theta)(x, \delta)\right)\right)^{\eta(x, \delta)}
\end{aligned}
$$


where the maps $M_{U}(\sigma): U \times[0, \infty) \rightarrow \Omega(U), M_{U}(\theta): U \times[0, \infty) \rightarrow \Omega(U)$ are given by $(9)$ with $\omega(\cdot, \cdot)$ equal to $\sigma(\cdot, \cdot)$ and $\theta(\cdot, \cdot)$, respectively.

Next, define the multimaps ext $\Delta:[a, b] \times C^{n}[a, b] \rightarrow \operatorname{comp}\left[\mathbb{R}^{n}\right], \operatorname{ext} \Phi:$ $C^{n}[a, b] \rightarrow \Pi\left[L^{n}[a, b]\right], \operatorname{ext} \Phi_{\varkappa, \nu}: U \times[0, \infty) \rightarrow \operatorname{cl}\left[L^{n}[a, b]\right]$ as follows

$$
\begin{gathered}
(\operatorname{ext} \Delta)(t, x)=\overline{\operatorname{ext}} \Delta(t, x), \\
(\operatorname{ext} \Phi)(x)=S((\operatorname{ext} \Delta)(\cdot, x)), \\
\left(\operatorname{ext} \Phi_{\varkappa, \nu}\right)(x, \delta)=\left((\operatorname{ext} \Phi)\left(M_{U}(\nu)(x, \delta)\right)\right)^{\varkappa(x, \delta)},
\end{gathered}
$$

here $\Delta:[a, b] \times C^{n}[a, b] \rightarrow \operatorname{comp}\left[\mathbb{R}^{n}\right]$ is the map generating the operator $\Phi:$ $C^{n}[a, b] \rightarrow \Pi\left[L^{n}[a, b]\right]$ (see $\left.(5)\right)$.

On a closed convex set $U \subset C^{n}[a, b]$ for every $\delta>0$ consider the inclusions

$$
\begin{gathered}
x \in \Psi_{\xi, \sigma}(x, \delta)+V \Phi_{\eta, \theta}(x, \delta), \\
x \in \Psi_{\xi, \sigma}(x, \delta)+V\left(\operatorname{ext} \Phi_{\varkappa, \nu}\right)(x, \delta),
\end{gathered}
$$

where the maps $\Psi_{\xi, \sigma}: U \times[0, \infty) \rightarrow \operatorname{cl}\left[C^{n}[a, b]\right], \Phi_{\eta, \theta}: U \times[0, \infty) \rightarrow \operatorname{cl}\left[L^{n}[a, b]\right]$, $\operatorname{ext} \Phi_{\varkappa, \nu}: U \times[0, \infty) \rightarrow \operatorname{cl}\left[L^{n}[a, b]\right]$ are defined by (31)-(35). We call inclusions $(36),(37)$ the inclusions with internal and external perturbations.

For each $\delta>0$ let $H_{\eta(\delta), \theta(\delta), \sigma(\delta), \xi(\delta)}(U)$, $\operatorname{ext} H_{\varkappa(\delta), \nu(\delta), \sigma(\delta), \xi(\delta)}(U)$ denote the sets of solutions for (36), (37), respectively belonging to $U \subset C^{n}[a, b]$.

Theorem 4. Let $U$ be a closed convex set of the space $C^{n}[a, b]$. Then for any $\xi(\cdot, \cdot), \eta(\cdot, \cdot), \varkappa(\cdot, \cdot) \in P\left(C^{n}[a, b] \times[0, \infty)\right)$ and $\sigma(\cdot, \cdot), \theta(\cdot, \cdot), \nu(\cdot, \cdot) \in \widetilde{P}\left(C^{n}[a, b] \times\right.$ $[0, \infty))$ the equalities

$$
H_{\mathrm{co}}(U)=\bigcap_{\delta>0} \overline{\operatorname{ext} H_{\varkappa(\delta), \nu(\delta), \sigma(\delta), \xi(\delta)}\left(U^{\delta}\right)}=\bigcap_{\delta>0} \overline{H_{\eta(\delta), \theta(\delta), \sigma(\delta), \xi(\delta)}\left(U^{\delta}\right)}
$$

take place, here $\overline{\operatorname{ext} H_{\varkappa(\delta), \nu(\delta), \sigma(\delta), \xi(\delta)}\left(U^{\delta}\right)}, \overline{H_{\eta(\delta), \theta(\delta), \sigma(\delta), \xi(\delta)}\left(U^{\delta}\right)}$ are the closures in the space $C^{n}[a, b]$ of solutions sets $\operatorname{ext} H_{\varkappa(\delta), \nu(\delta), \sigma(\delta), \xi(\delta)}\left(U^{\delta}\right), H_{\eta(\delta), \theta(\delta), \sigma(\delta), \xi(\delta)}\left(U^{\delta}\right)$, respectively.

Proof. We show first that

$$
H_{\mathrm{co}}(U) \subset \bigcap_{\delta>0} \overline{\operatorname{ext} H_{\varkappa(\delta), \nu(\delta), \sigma(\delta), \xi(\delta)}\left(U^{\delta}\right)} .
$$

Let $x \in H_{\mathrm{co}}(U)$ and show that for each $\delta>0, x$ is a limit point of the set $\operatorname{ext} H_{\varkappa(\delta), \nu(\delta), \sigma(\delta), \xi(\delta)}\left(U^{\delta}\right)$. Indeed, since for any $y \in C^{n}[a, b]$ the equality $\overline{\mathrm{co}}((\operatorname{ext} \Phi)(y))=\overline{\mathrm{co}}(\Phi(y))$ holds (see [10]), then, according to Theorem 1, $x$ is a quasisolution for inclusion (1), in which $\Phi(\cdot)=\operatorname{ext} \Phi(\cdot)$ (the map $\operatorname{ext} \Phi$ : $C^{n}[a, b] \rightarrow \Pi\left[L^{n}[a, b]\right]$ is defined by $\left.(33),(34)\right)$. This means that there exist an element $v \in \Psi(x)$ and a sequence $z_{i} \in \operatorname{ext} \Phi(x), i=1,2, \ldots$, such that for every $i=1,2, \ldots$ we have the relation $x_{i}=v+V z_{i} \in U^{\delta}$ and $x_{i} \rightarrow x$ in $C^{n}[a, b]$ as $i \rightarrow \infty$.

Next, prove that there exists a number $I$ such that for every $i \geq I$

$$
x_{i} \in \operatorname{ext} H_{\varkappa(\delta), \nu(\delta), \sigma(\delta), \xi(\delta)}\left(U^{\delta}\right) .
$$


Since $\nu(\cdot, \cdot), \sigma(\cdot, \cdot) \in \widetilde{P}\left(C^{n}[a, b] \times[0, \infty)\right)$, one can find a number $i_{0}$ such that for all $i \geq i_{0}$ the inclusions $x \in B_{C^{n}[a, b]}\left[x_{i} ; \nu\left(x_{i}, \delta\right)\right], x \in B_{C^{n}[a, b]}\left[x_{i} ; \sigma\left(x_{i}, \delta\right)\right]$ are true. This means that for each $i \geq i_{0} x \in M_{U^{\delta}}(\nu)\left(x_{i}, \delta\right)$ and $x \in M_{U^{\delta}}(\sigma)\left(x_{i}, \delta\right)$, where the multimaps $M_{U^{\delta}}(\nu)(\cdot, \cdot), M_{U^{\delta}}(\sigma)(\cdot, \cdot)$ are defined by $(9)$ in which $U=U^{\delta}$, $\omega=\nu$ and $\omega=\sigma$, respectively. Then for every $i \geq i_{0}$ the following inclusions are true

$$
\Psi(x) \subset \Psi_{\xi, \sigma}\left(x_{i}, \delta\right), \quad(\operatorname{ext} \Phi)(x) \subset \operatorname{ext} \Phi_{\eta, \nu}\left(x_{i}, \delta\right) .
$$

From (41) and the definition of inclusion (37) it follows that for any $i \geq I=i_{0}$ we get (40), and this means that $x$ is a limit point of the set $\operatorname{ext} H_{\varkappa(\delta), \nu(\delta), \sigma(\delta), \xi(\delta)}\left(U^{\delta}\right)$, i.e., $x \in \overline{\operatorname{ext} H_{\varkappa(\delta), \nu(\delta), \sigma(\delta), \xi(\delta)}\left(U^{\delta}\right)}$ and, consequently, relation (39) takes place.

The embedding

$$
\bigcap_{\delta>0} \overline{\operatorname{ext} H_{\varkappa(\delta), \nu(\delta), \sigma(\delta), \xi(\delta)}\left(U^{\delta}\right)} \subset H_{\mathrm{co}}(U)
$$

can be proved by an analogy with inclusion (22) in the proof of Theorem 2 .

The second equality in (37) is proved in an analogous manner.

Remark 8. So, in the presence of internal perturbations in the right-hand side of the inclusion no "average" accuracy in calculating the values of the map $\Phi: C^{n}[a, b] \rightarrow \Pi\left[L^{n}[a, b]\right]$ can guarantee "restoring" the set $\overline{H(U)}$ by the closures in the space $C^{n}[a, b]$ of the sets of approximate solutions. This is possible only in the case where the density principle holds.

Remark 9. Note that the general definition of the concept of approximate solution ( $\delta$-solution) for differential inclusion with convex and upper semicontinuous right-hand side was introduced by A. F. Filippov (see [12]). This definition plays a crucial role in studying differential inclusions, for it satisfies the fundamental property: the limit of converging approximate solutions is also a solution. Here, for studying the asymptotic properties of the sets of approximate solutions for inclusion (1) with right-hand side not necessarily closed- and convex-valued, we use (see inclusions (16), (36), (37)) slightly different definitions of approximate solutions. The difference between our definitions and the one given by Filippov is that the values of multimaps "describing approximate functional inclusions" (see (16),(36),(37)) are "not to be convexified".

\section{ACKNOWLEDGEMENT}

The work is partially supported by RFBR (grant 07-01-00305) and carried out in the framework of the Item No 1.6.07 of the Thematic Research Plan of Tambov State University.

\section{REFERENCES}

1. A. I. Bulgakov, O. P. Belyaeva, and A. A. Grigorenko, On the theory of perturbed inclusions and its applications. (Russian) Mat. Sb. 196(2005), No. 10, 21-78; English transl.: Sb. Math. 196(2005), No. 9-10, 1421-1472. 
2. A. I. Bulgakov and L. I. TKaCh, Some results on the theory of perturbations of multioperators with closed convex values by a map of Hammershtein type with nonconvex values and their applications. Vestnik Tambov State Univ., Ser. Natural and Technical Sciences 2(1997), No. 2, 111-120.

3. A. I. Bulgakov and L. I. TKach, Asymptotic representation of the sets of $\delta$-solutions for the Hammershtein type inclusion. Vestnik Tambov State Univ., Ser. Natural and Technical Sciences 2(1997), No. 3, 294-298.

4. A. I. Bulgakov and L. I. TKACH, Perturbation of a convex-valued operator by a Hammerstein-type multivalued mapping with nonconvex images, and boundary value problems for functional-differential inclusions. (Russian) Mat. Sb. 189(1998), No. 6, 332; English transl.: Sb. Math. 189(1998), No. 5-6, 821-848.

5. A. I. Bulgakov and L. I. TKach, Perturbation of a single-valued operator by a multivalued mapping of Hammerstein type with nonconvex images. (Russian) Izv. Vyssh. Uchebn. Zaved. Mat. 1999, No. 3, 3-16; English transl.: Russian Math. (Iz. VUZ) 43(1999), No. 3, 1-13.

6. A. D. Ioffe and V. M. Tikhomirov, Theory of extremal problems. (Russian) Series in Nonlinear Analysis and its Applications, Nauka, Moscow, 1974.

7. A. I. Bulgakov, A functional-differential inclusion with an operator that has nonconvex images. (Russian) Differentsial'nye Uravneniya 23(1987), No. 10, 1659-1668.

8. I. T. Kiguradze, Boundary value problems for systems of ordinary differential equations. (Russian) Itogi Nauki i Tekhniki, Current problems in mathematics. Newest results, Vol. 30 (Russian), 3-103, 204, Akad. Nauk SSSR, Vsesoyuz. Inst. Nauchn. i Tekhn. Inform., Moscow, 1987; English transl.: J. Soviet Math. 43(1988), No. 2, 2259-2339.

9. I. T. Kiguradze, Some singular boundary value problems for ordinary differential equations. (Russian) Izdat. Tbilis. Univ., Tbilisi, 1975.

10. A. I. Bulgakov, Integral inclusions with nonconvex images and their applications to boundary value problems for differential inclusions. (Russian) Mat. Sb. 183(1992), No. 10, 63-86; English transl.: Russian Acad. Sci. Sb. Math. 77(1994), No. 1, 193-212.

11. A. Pliś, Trajectories and quasitrajectories of an orientor field. Bull. Acad. Polon. Sci. Sér. Sci. Math. Astronom. Phys. 11(1963), 369-370.

12. A. F. Filippov, Differential equations with discontinuous right-hand sides. (Russian) Nauka, Moscow, 1985.

13. A. I. Bulgakov and V. V. Skomorokhov, Approximation of differential inclusions. (Russian) Mat. Sb. 193(2002), No. 2, 35-52; English transl.: Sb. Math. 193(2002), No. 1-2, 187-203.

14. A. F. Filippov, Classical solutions of differential equations with the right-hand side multi-valued. (Russian) Vestnik Moskov. Univ. Ser. I Mat. Mekh. 22(1967), No. 3, 1626.

15. V. I. Blagodatskikh and A. F. Filippov, Differential inclusions and optimal control. (Russian) Topology, ordinary differential equations, dynamical systems. Trudy Mat. Inst. Steklov. 169(1985), 194-252.

16. A. V. Arutyunov, S. M. Aseev, and V. I. Blagodatskikh, Necessary conditions of the first order in a problem of the optimal control of a differential inclusion with phase constraints. (Russian) Mat. Sb. 184(1993), No. 6, 3-32; English transl.: Russian Acad. Sci. Sb. Math. 79(1994), No. 1, 117-139.

17. A. V. Arutyunov, Extremum conditions. Abnormal and degenerate problems. Mathematics and its Applications. (Russian) Faktorial, Moscow, 1997.

18. E. S. Polovinkin, Theory of multi-valued maps. MFTI, Moscow, 1982. 
19. A. E. Irisov and E. L. Tonkov, On the closure of the set of periodic solutions of a differential inclusion. (Russian) Differential and integral equations (Russian), 32-38, 134, Gor'kov. Gos. Univ., Gorki, 1983.

20. A. I. Bulgakov, Continuous branches of multivalued mappings, and integral inclusions with nonconvex images and their applications. I, II, III. (Russian) Differentsial'nye Uravneniya 28(1992), No. 3, 4, 5, 371-379, 566-571, 739-746; English transl.: Differential Equations 28(1992), No. 3, 4, 5, 303-311, 454-458, 587-592.

21. A. I. Bulgakov, A. A. Efremov, and E. A. Panasenko, To the issue of stability of differential inclusions. Vestnik Tambov State Univ., Ser. Natural and Technical Sciences 4(1999), No. 4, 461-470.

(Received 10.01.2007; revised 6.04.2007)

Authors' addresses:

A. Bulgakov and A. Korobko

G. R. Derzhavin Tambov State University

Department of Algebra and Geometry

Internazional'naya str., 33

Tambov 392000, Russia

E-mails: aib@tsu.tmb.ru

prof13@yandex.ru

A. Grigorenko

Moscow State Forest University

MSFU, 1-st Institutskaya, 1,

Mytishchy-5, Moscow oblast 141005, Russia

E-mail: aib@tsu.tmb.ru 